\title{
Supply Chain Reactivity Assessment Regarding Two Negotiated Commitments: Frozen Horizon and Flexibility Rate
}

\author{
Aïcha Amrani-Zouggar, Jean-Christophe Deschamps, and Jean-Paul Bourrières
}

\author{
IMS - LAPS/GRAI, University of Bordeaux, UMR 5218CNRS \\ 351, Cours de la libération, 33405 Talence Cedex, France \\ \{aicha.amrani, jean-christophe.deschamps, \\ jean-paul. bourrieres\} aims-bordeaux.fr
}

\begin{abstract}
The problem addressed in this paper is the supply chain reactivity assessment through numerical experimentations regarding specifically two negotiated commitments within supply contract: the frozen horizon and flexibility rate. Analysis of impact of these commitments on each partner will be depicted in term of storage costs, reliability and reactivity indicators. The decision making inside each partner is operated under rolling horizon planning and based on production linear programming model wherein different contractual commitments are included. To carry out experimental scenarios, simulation platform is developed from which expecting numerical results afford deciders to get more understanding about the commitments that should be contracted and the relevant dimensioning of them.
\end{abstract}

Keywords: Supply chain, Tactical production planning, supply contract commitments, simulation.

\section{Introduction}

In increasingly competitive context where the customer's requirements are still growing and the demand is highly volatile, many risks are threatening the order fulfilment process. One way to cope with these risks is to extend the partner's network that brings some stability to the network. The deciders are aware that collaborative approaches constitute a strong axis of reducing surrounding risks. However, if the network may constitutes a response to some risks, it generates other ones [1] therefore it becomes essential to achieve the challenging aim of ensuring whole supply chain performance. Our current work is based on collaborative relationships that are framed by supply contracts among the partners. Indeed, contractual agreement of supplying products from the suppliers if accurately designed may provide the required enhancement of performance. Negotiate specific clauses of collaboration with suppliers through contract commitments becomes an excellent coordination mechanism [2] stabilizing the relationship over certain horizon of time and minimizing industrial risks (such as high inventory level, backorders, delay of deliveries, unsatisfying customer and rush orders). 
In our previous works, we have shown in [3] the intrinsic relationship between risks and the negotiated clauses. Further, we have revealed through simulation an effective quantified sensitivity of buyer and supplier performance to different quantity commitments contracts [4]. In this paper, our proposition focuses on the analysis of supply chain reactivity regarding two negotiated commitments: Frozen horizon and flexibility rate. The purpose of this study is beyond the simple checking of sensitivity leading to show explicitly to which partner do the commitment benefit and how explain it?

The remainder paper is organized as follows. The related work of supply contracts is presented in Section 2. In Section 3, description of tactical production planning model is provided with an overview of contractual agreements (frozen horizon and flexibility rate) subjected to the study. Section 4 is devoted to discussing the reactivity of supply chain regarding two evoked commitments. The analysis is done inside a buyer-supplier relationship. In Section 6, further experimentations come to reinforce our contributions and conclusion comes to end this paper.

\section{Literature Review}

Great attention is granted to collaborative approaches which are widely studied. Supply contracts constitute part of them and consist in agreements between buyer and supplier, that aim at protect their respective own and joint interests, describe the limits of risk, enounce terms and conditions of fulfilment. Once clauses clearly identified, monitoring of order fulfilment process becomes possible at the operational level. In literature, we distinguish for example [5], [6] where the authors aim through their research to get optimal purchasing policy. We notice that majority of works are optimization models under contractual context based on stochastic modelling [7] or continuous modelling [8]. Our research is quite different in term of problem modelling and objective, in one hand we propose a discrete planning model (like [9]) rather infrequently used in this field. In the other hand, the simulation that will be carried out is the basis of quantitative analysis to oversee simultaneous impact on performance of varying two commitments.

The aim of our work is rather to check the impact of each commitment (frozen horizon and flexibility rate) on own and overall performance than to assess the numeric value of the optimisation.

\section{Tactical Production Planning Model}

Each actor of considered supply chain SC is assimilated to a DMU (Decision Making Unit) with carrying out the calculation of production, replenishment and delivery plans and the levels of necessary stocks to each period. The model concerns multi-product (p), multi periods ( $\mathrm{t}$ ), with finished nominal capacity that accommodates adjustment by additional capacity represented here by the decision to resort to overtime (additional hours). Planning process is dynamically depicted according to the principle of rolling horizon planning. Any modification that occurs either on the demand or on operational execution can be taken into account at the next planning occurrence. 
We make the assumption that Demand is deterministic and known along the planning horizon. The production planning process developed is based on linear programming modelling with a criterion to maximize under a set of constraints.

\subsection{Model}

The table 1 summarizes various notations adopted to formalize the production planning process.

Table 1. Notation list

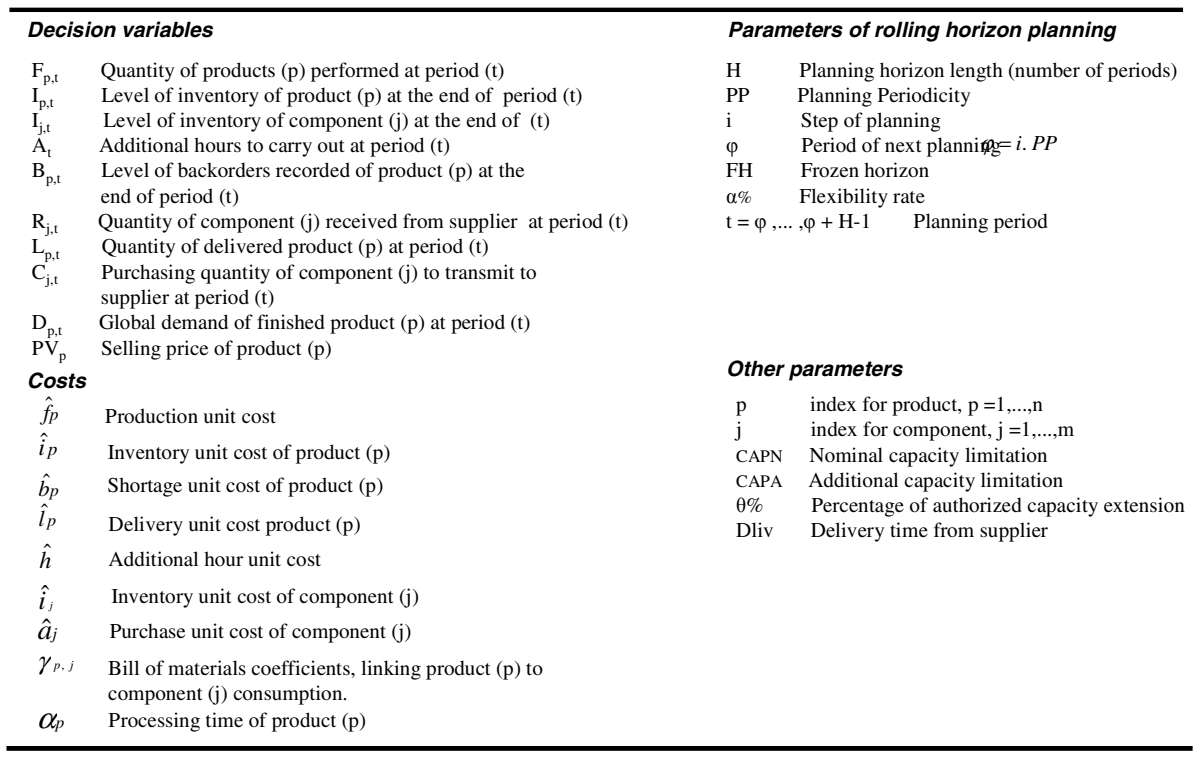

The objective function (1) tends to maximize the profit resulting from the difference between the sales and the various undergoing costs:

$\max \sum_{t=\varphi}^{\varphi+H-1}\left(\sum_{p=1}^{n} P V_{p} \cdot L_{p, t}-\left(\hat{f}_{p} \cdot F_{p, t}+\hat{i}_{p} \cdot I_{p, t}+\hat{b}_{p} \cdot B_{p, t}+\hat{l}_{p} \cdot L_{p, t}-\hat{h} \cdot A_{t}\right)-\left(\sum_{j=1}^{m} \hat{i}_{j} I_{j, t}+\hat{a}_{j} \cdot R_{j, t}\right)\right)$

Under the constraints

$$
\begin{aligned}
& I_{p, t}=I_{p, t-1}+F_{p, t}-L_{p, t} \\
& B_{p, t}=B_{p, t-1}+D_{p, t}-L_{p, t} \\
& I_{j, t}=I_{j, t-1}+R_{j, t}-\sum_{p=1}^{n} \gamma_{p, j} . F_{p, t} \\
& \sum_{p=1}^{n} \alpha_{p .} F_{p, t} \leq C A P N+A_{t} \\
& A_{t} \leq C A P A \mid C A P A=\theta \% . C A P N
\end{aligned}
$$$$
\forall p=1, \ldots, n
$$$$
\forall p=1, \ldots, n
$$$$
\forall p=1, \ldots, n, \forall j=1, \ldots, m
$$$$
\forall p=1, . ., n
$$$$
\forall p=1, \ldots, n
$$

These constraints are valid $\forall t=\varphi, \ldots, \varphi+H-1$.

The equation (2) is a quantity conservation equation; it links the level of stocks and the produced quantity. The equation (3) links the level of shortages to the demand and 
deliveries. The equation (4) calculates the level of stocks of components according to the receptions, the production and bill of materials coefficients. The constraint (5) gives the limits of production quantities according to the normal capacity and additional capacity. The constraint (6) guarantees limitation of overtime in such way that additional capacity is equal to a certain percentage $(\theta \%)$ of the normal capacity.

\subsection{Contractual Constraints}

The specificity of this contribution is to check the potential impact on reactivity of partners when varying two commitments negotiated during the design of supply contract. In this section we introduce more deeply these clauses and formalize them in order to integrate them into the production planning process.

\subsubsection{Frozen Horizon (FH) and Flexibility Rate ( $\alpha \%)$}

In fluctuant demand context, rolling horizon planning often comes to palliate the uncertainty impact giving the possibility to the deciders to update their planning process including new information (demand fluctuation, delivery disturbance...). However, the industrial reality shows the necessity to stabilize the activities during certain amount of time called "frozen horizon" (FH) in [10], or "freezing time". When a frozen horizon is negotiated through supply contract between two adjacent partners, the value of purchasing quantities over frozen horizon have a commitment values and could not be modified with next planning occurrence. Over a frozen horizon, a rate of flexibility $(\alpha \%)$ is negotiated to enable the buyer to modify demand among two planning occurrences (Fig.1). Flexibility rate concept has been studied in literature. These are some definitions founded: for [7], it is an upside and downside flexibility bounds of order quantity. Bassok \& Anupindi in [8] considers a model in which the amount of increase or reduction in the order for a given period is limited to a percentage of the current order for the period. We notice that these definitions converge to the same idea, flexibility rate $(\alpha \%)$ consists in two bounds allowing adaptation to the uncertainty of the market.

These commitments constitute a kind of constraints that each partner has to model, to formalize and take into account during each occurrence of the planning process. Our work consists on modelling these commitments and introduces them in a tactical planning model. Over seven periods of FH in (Fig.1), the producer starts a certain production volume. Indeed, the necessary purchasing quantities $C^{i}{ }_{j, t}$ from supplier for executing orders will have a commitment value. They could not be modified at next planning moments.

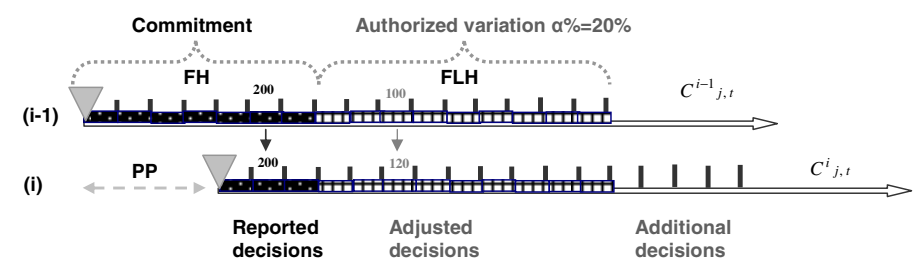

Fig. 1. Frozen horizon and flexibility rate 
Beyond the frozen horizon of the producer, we find the flexible horizon (FLH) which is by definition less rigid. On this horizon the producer is approved to vary his orders of over or under certain percentage $(\alpha \%)$ at the next planning occurrence (example: $\alpha \%=20 \%$ means from 100 units to 120 units). After planning period PP, additional decisions come to complete the length of horizon.

We claim that it should be taken care that the quantity of purchasing orders $\left(\mathrm{C}_{\mathrm{j}, \mathrm{t}}\right)$, belongs to the intervals authorized by negotiated $(\alpha \%)$. Constraints of this context may be written as follows:

$$
\begin{aligned}
& R^{i}{ }_{j, t}=C^{i}{ }_{j,(t-\text { Dliv })} \\
& F^{i}{ }_{p, t}=F^{i-1}{ }_{p, t} \\
& A t^{i}=A t^{i-1}{ }^{i-1}{ }_{j, t}=C^{i-1}{ }_{j, t} \\
& C^{i-1}{ }_{j, t}(1-\alpha \%) \leq C^{i}{ }_{j, t} \leq C^{i-1}{ }_{j, t} .(1+\alpha \%)
\end{aligned}
$$

$$
\begin{aligned}
& \forall t=\varphi, \ldots, \varphi+H-1 \\
& \forall t=\varphi, . ., \varphi+(F H-P P-1) \\
& \forall t=\varphi, . ., \varphi+(F H-P P-1) \\
& \forall t=\varphi, . ., \varphi+(F H-P P-1) \\
& \forall t=\varphi+F H-1, \ldots, \varphi+F L H-1
\end{aligned}
$$

To ensure the arrivals of $\left(\mathrm{R}_{\mathrm{j}, \mathrm{t}}\right)$ the purchasing quantity $\left(\mathrm{C}_{\mathrm{j}, \mathrm{t}}\right)$ have to be transmitted to supplier some periods $\left(\mathrm{D}_{\mathrm{liv}}\right)$ in advance; it is expressed through equation (7). Equations (8), (9), (10) express the report of the frozen decisions. For example, Produced quantities $\mathrm{F}_{\mathrm{p}, \mathrm{t}}^{\mathrm{i}}$ at step (i) will be assigned the value of previous produced quantities $\mathrm{F}^{\mathrm{i}-}$ $1_{\mathrm{p}, \mathrm{t}}$ of previous planning step as long as $t=\varphi, \ldots, \varphi+(F H-P P-1)$. See Fig.1: At planning step (i-1), we report decisions of $\left(\mathrm{C}_{\mathrm{j}, \mathrm{t}}^{\mathrm{i}-1}\right)$ over periods $5,6,7$. The constraints (11) expresses the flexibility commitment, as long as $t=\varphi+F H-1, \ldots, \varphi+F L H-1$, the orders transmitted at planning step (i) $\mathrm{C}_{\mathrm{j}, \mathrm{t}}^{\mathrm{i}}$ might vary within the interval plus or less $(\alpha \%)$ from a previous order $\mathrm{C}_{\mathrm{j}, \mathrm{t}}^{\mathrm{i}-\mathrm{e}}$.

\section{Experiments and Analysis}

The simulation platform dedicated to perform different scenarios has been detailed in our previous contribution [4]. In this experimental section, based on our simulation platform, we test different configurations of flexibility rate, and frozen horizon between a buyer OEM and supplier PRODU. The results will be expressed in terms of different performance indicators, as the profit (criterion to maximize), the costs of adaptation (including finished product inventory costs, delivered components inventory costs, backorders and additional hours costs); the reactivity is then expressed by the number of required periods by the partner to satisfy the entire demand and the reliability is calculated as the ratio of real delivery and expected delivery products. These indicators allow a better understanding of supply chain behaviour according to the point of view of decision maker. At first, buyer supplier view is analyzed in order to give off global trends. To depict accurately the impact of parameters on both partners, more focused analysis will be carried out. Demand disturbance would be tested at period $\mathrm{t}=11$ with enhancement of 600 products.

\subsection{Buyer-Supplier View: Global Trends}

Global trend shows (Table 2.) that the supply chain behaves differently according to the adopted configuration of FH and $\alpha \%$. More the FH is high better it is for supplier 
(PRODU) but not to the buyer. More the flexibility rate increases, better it is for the buyer (OEM) but unfortunately not so benefit for supplier PRODU. Further, we achieve a detailed analysis on each partner to perceive the mechanism that leads the partner to record these performances.

Table 2. Buyer \& supplier performance trend

\begin{tabular}{|c|c|c|c|c|c|c|c|c|c|}
\hline & \multicolumn{3}{|c|}{$\mathrm{FH}=6$} & \multicolumn{3}{|c|}{$\mathrm{FH}=7$} & \multicolumn{3}{|c|}{$\overline{F H}=8$} \\
\hline & OEM & PRODU & Overall & OEM & PRODU & Overall & OEM & PRODU & Overall \\
\hline \multicolumn{10}{|l|}{$\alpha=13 \%$} \\
\hline Profit (\$) & 2038071 & 817440 & 2855512 & 2033776 & 820402 & 2866602 & 2023002 & 825112 & 2848114 \\
\hline Costs (\$) & 40679 & 23359 & 64039 & 46287 & 20398 & 66688 & 58465 & 15688 & 74152 \\
\hline Reactivity (period) & 4.00 & 3.00 & & 6.00 & 2.00 & & 7.00 & 3.00 & \\
\hline Reliability (\%) & 98.18 & 99.51 & & 97.48 & 99.69 & & 96.06 & 99.68 & \\
\hline \multicolumn{10}{|l|}{$\alpha=20 \%$} \\
\hline Profit (\$) & 2045910 & 815802 & 2861712 & 2045424 & 816644 & 2871616 & 2045262 & 816557 & 2861819 \\
\hline Costs (\$) & 40341 & 24998 & 65339 & 40341 & 24155 & 64496 & 40503 & 24243 & 64746 \\
\hline Reactivity (period) & 4.00 & 2.00 & & 4.00 & 2.00 & & 4.00 & 2.00 & \\
\hline Reliability (\%) & 98.13 & 99.61 & & 98.13 & 99.61 & & 98.11 & 99.64 & \\
\hline \multicolumn{10}{|l|}{$\alpha=35 \%$} \\
\hline Profit (\$) & 2051684 & 81689 & 2868578 & 2051198 & 817737 & 2868934 & 2049457 & 821106 & 2871868 \\
\hline Costs $(\$)$ & 36001 & 34705 & 70706 & 36000 & 33863 & 69864 & 37694 & 30493 & \\
\hline Reactivity (period) & 4.00 & 1.00 & & 4.00 & 1.00 & & 4.00 & 1.00 & \\
\hline Reliability (\%) & 98.13 & 99.69 & & 98.13 & 99.69 & & 98.13 & 99.69 & \\
\hline
\end{tabular}

According to these results, we may notice that these two clauses are antagonists. That's why it becomes important to relevantly negotiate them. Another interesting observation that simulation shows is that a compromise of performance (by regulating two parameters) could be obtained to achieve expected performance. It doesn't mean that maximum value of flexibility only achieve accepted performance. Configuration of $(\alpha=20 \%, \mathrm{FH}=8)$ records less overall costs than configuration $(\alpha=35 \%, \mathrm{FH}=7)$ it underlines the fact that the best configuration of commitments compromises can be obtained by simulation and the best configurations aren't particularly obviously expected.

\subsection{Analysis of Local Performance Per Partner}

What happens with varying frozen horizon, why is it benefiting for supplier?

We highlight the undertaken points to make our analysis; it consists on observing the costs of inventory levels, backorders levels and required additional hours. Let us consider the scenario where flexibility between two partners is equal to $13 \%$; we focus on the differences of costs generated by two configurations of frozen horizon $\mathrm{FH}=6$ and $\mathrm{FH}=7$. (Fig.2)

The observation shows that for OEM more frozen horizon is high, more he will be constrained to freeze also his production planning according to the arriving components from suppliers, less periods remains for performing the smooth of the load so less production could be done. $(\mathrm{FH}=7)$ generates by this way more backorders, huge costs and smaller reactivity. FH seems not to be benefit to the buyer at all, it prevents 

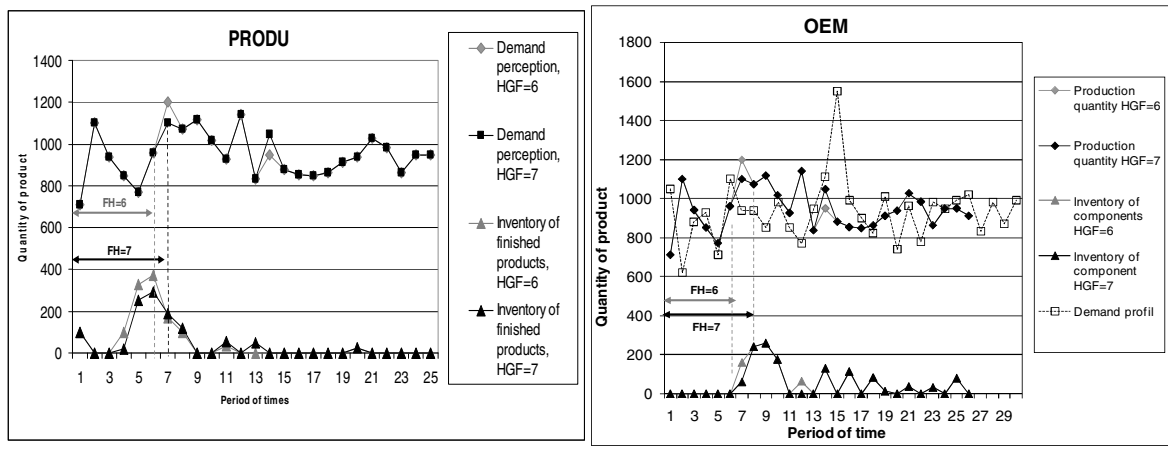

Fig. 2. Profiles with varying FH at producer (OEM) and supplier (PRODU)

him from smoothing and do not allows the transmission of the real demand changing, limited to only what supplier would like to perceive. Whereas for PRODU, more the frozen horizon is high $(\mathrm{FH}=7)$ better it is, the supplier hasn't ever to perceive the demand pick, he considers previously planned production and is not obliged to make high inventory of ended product (as it is the case for $\mathrm{FH}=6$ ). Indeed, if frozen horizon decreases, (Fig.2) shows the necessity to anticipate by preparing more inventory level generating high adaptation costs for supplier.

What happens with varying flexibility rate, why is it benefiting for buyer?
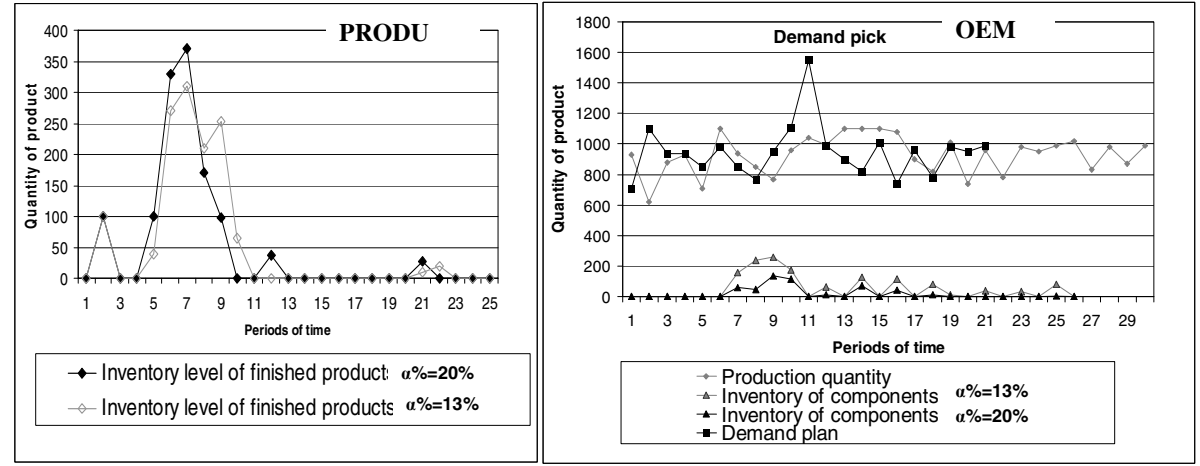

Fig. 3. Profiles with varying $\alpha \%$ at buyer (OEM) and supplier (PRODU)

We carry out an analysis in scenario with $(\alpha=13 \%, \mathrm{FH}=6)$ and $(\alpha=20 \%, \mathrm{FH}=6)$ configurations of commitments. The results enable us to show that increased $\alpha \%$ granted by the supplier benefits to OEM and penalise PRODU.

In (Fig.3), we may notice with increasing $\alpha \%$ that inventory level of components at OEM decreases. In deed, with increasing flexibility rate, more deliveries can be expected avoiding cumulating inventory stocks at the buyer OEM. Whereas at the supplier, more inventories of finished products becomes required to be reactive to buyer pick; therefore, we see that the smooth of inventory load becomes bigger in scenario $\alpha=20 \%$ configuration. 


\section{Conclusion and Perspectives}

Starting from simulation study wherein we check for the impact of $\mathrm{FH}$ and $\alpha \%$ commitments, we can release some interesting observations about their relevance and their usefulness at each partner of supply chain. More the flexibility is high, less inventory at the buyer are required enhancing by the way his performance. More the FH is high better it is for the supplier who avoids perceiving the demand variation and is not obliged to make high inventory level. In this paper, the supply chain is still reduced to its elementary network (producer and supplier). The simulation platform enables us to carry out other simulations extended to 3 partners in the network. These results are under finalizing and will be published in future work. Converging to adequate contract between SC partners still remains a delicate step hard to achieve in industrial reality. This study wishes to provide deciders with more visibility about the commitments able to protect them or on contrary threat their interests in order to guide them to thoroughly consider them during negotiation process.

\section{References}

1. Christopher, M.: Logistics and Supply Chains management. In: Financial Times. Prentice Hall, Englewood Cliffs (2005)

2. Mentzer, J.T., Foggin, J.H., Golicic, S.G.: Supply chain collaboration: enablers, impediments and benefits. Supply Chain Management Review 4(4), 52-58 (2000)

3. Amrani-Zouggar, A., Deschamps, J.C., Bourrières, J.P.: Supply chain contracts design according to industrial risks. In: IFIP-APMS Proceedings, pp. 22-33 (2008)

4. Amrani-Zouggar, A., Deschamps, J.C., Bourrières, J.P.: Supply chain planning under various quantity commitment contracts. In: Proceedings of IFAC - INCOM (2009)

5. Cachon, G.: Supply chain coordination with contracts. In: Handbooks in Operations Research and Management Science: Supply Chain Management. Steve Graves and Ton de Kok. North Holland, Amsterdam (2003)

6. Bassok, Y., Anupindi, R.: Analysis of supply contracts with total minimum commitments. IIE Transactions 29(5), 373-381 (1997)

7. Liston, P., Byrne, P.J., Heavey, C.: Contract costing in outsourcing enterprises: Exploring the benefits of discrete-event simulation. Int. J. Production Economics 110, 97-114 (2007)

8. Anupindi, R., Bassok, Y.: Supply contracts with quantity commitments and stochastic demand. In: Tayur, S., Ganeshan, R., Magazine, M. (eds.) Quantitative models for supply chain management, pp. 198-232. Kluwer Academic Publishers, Dordrecht (1999)

9. Schneeweiss, C., Zimmer, K., Zimmermann, M.: The design of contracts to coordinate operational interdependencies within the supply chain. Int. J. Production Economics 92, 4359 (2003)

10. Millart, H.H.: The impact of rolling horizon planning on the cost of industrial fishing activity. Computers Operational Research 25(10), 825-837 (1998) 